\title{
PELÍCULAS CONDUCTORAS DE ALMIDÓN DE YUCA (CASSAVA) COMO MATERIAL PARA UN ACUMULADOR ELECTROQUÍMICO DE CARGA (BATERÍA)
}

\author{
Álvaro Arrieta Almario*a, Andrés Jaramillo Muñoz ${ }^{\mathrm{b}}$, Manuel Palencia Luna ${ }^{\mathrm{c}}$
}

\begin{abstract}
RESUMEN
En la actualidad, existe la necesidad de desarrollar nuevos dispositivos capaces de almacenar energía, que sean ligeros, flexibles y amigables con el medio ambiente. En este trabajo se presenta la síntesis y caracterización de películas delgadas a base de almidón de yuca (cassava) y películas delgadas de polipirrol; las cuales fueron utilizadas en el desarrollo de un acumulador electroquímico. Las películas de almidón fueron preparadas a partir de almidón nativo de yuca y la adición de plastificantes y perclorato de litio. Por otro lado, las películas de polipirrol (PPy) fueron dopadas con Índigo Carmín (IC) y ácido p-toluensulfónico (pTS) y se sintetizaron electroquímicamente en una celda electroquímica de tres electrodos (trabajo, referencia y auxiliar). La caracterización se llevó a cabo empleando las técnicas electroquímicas de voltametría cíclica (VC) y espectroscopía de impedancia electroquímica (EIE). Los resultados indican un buen comportamiento electroquímico de las películas delgadas de almidón, con conductividades aproximadas de $3 \times 10^{-3} \mathrm{~S} \mathrm{~cm}^{-1}$ y una buena actividad rédox que permite la acumulación de carga en el dispositivo armado.
\end{abstract}

Palabras clave: Batería polimérica, electrodos de polipirrol, almidón de yuca.

\section{CONDUCTIVE FILMS FROM CASSAVA STARCH AS MATERIAL FOR AN ELECTROCHEMICAL ACCUMULATOR (BATTERY)}

\begin{abstract}
Currently, there is a need to develop new devices capable of storing energy, which are lightweight, flexible and friendly to the environment. In this paper we present the synthesis and characterization of cassava starch films and polypyrrole films, which were used in the development of an electrochemical battery. The starch films were synthesizedchemically from native cassava starch adding plasticizers and lithium perchlorate. Moreover, polypyrrole films were doped with p-toluenesulfonic acid (pTS) and Indigo Carmin (IC) and synthesized electrochemically in an electrochemical cell of three electrodes (working, reference and auxiliary). The characterization was carried out using electrochemical techniques of cyclic voltammetry $(\mathrm{CV})$ and electrochemical impedance spectroscopy (EIS). The results indicate excellent electrochemical behavior of starch films with conductivities of approximately

\footnotetext{
*a Departamento de Biología y Química ,Facultad de Educación y Ciencias, Universidad de Sucre, Kra 28 No. 5- 267 Barrio Plaza Roja, Sincelejo- Colombia. e-mail: alvaroangel.arrieta@gmail.com

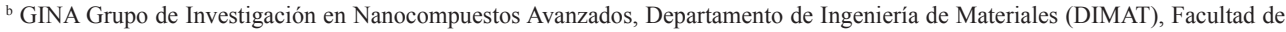
Ingeniería (FI), Universidad de Concepción (UdeC), Edmundo Larenas 270 (exterior), Casilla 160-C, Concepción, Chile.

c Departamento de Química, Facultad de Ciencias Naturales y Exactas, Universidad del Valle, Cali-Colombia.
} 
$3 \times 10^{-3} \mathrm{~S} \mathrm{~cm}^{-1}$ and a good redox activity which allow the accumulation of charge in the device assembly.

Key words: Polymeric battery, polypyrrole electrodes, starch films.

\section{INTRODUCCIÓN}

Como una manera de contribuir con el desarrollo de tecnologías limpias e independizarse de las fuentes petroquímicas, se está llevando a cabo investigaciones encaminadas hacia la producción y almacenamiento de energía con fuentes alternativas y utilizando nuevos materiales biodegradables, además de mejorar el futuro energético y los problemas de contaminación que ocasionan la producción de energía ${ }^{1}$. En este sentido podemos ver que la tecnología de las baterías tiene un futuro promisorio en el camino hacia la sostenibilidad, siendo el desarrollo de baterías poliméricas, las que recientemente han ganado mayor interés por la comunidad científica y la industria, principalmente por la posibilidad de fabricar dispositivos flexibles y amigables con el medio ambiente ${ }^{1,2}$.

Es común utilizar el almidón en la elaboración de biopelículas para empaques comestibles, pero han surgido también investigaciones encaminadas hacia el desarrollo y estudio de las propiedades conductoras que este material puede llegar a adquirir ${ }^{3,4}$. Los estudios que se han hecho como biopolímero conductor (conductor iónico sólido) han atraído la atención porque se obtienen propiedades eléctricas y mecánicas favorables para su utilización como material conductor y semiconductor y además, porque el almidón es un polímero natural, abundante, renovable, económico y biodegradable ${ }^{5}$.

La conducción en este biopolímero se debe a la movilidad iónica; es decir, el transporte de carga tiene lugar mediante el salto de los iones a posiciones vecinas accesibles en la estructura del material ${ }^{6}$, y es esta capacidad de conducir la electricidad, lo que hace a los biopolímeros conductores muy atractivos para el desarrollo de nuevas tecnologías, es el caso de los acumuladores electroquímicos de $\operatorname{carga}^{7,8}$.

El propósito principal por el cual se ha llevado a cabo este trabajo, es desarrollar un acumulador electroquímico de carga, empleando como medio electrolítico sólido un polímero conductor de almidón de yuca y películas de polipirrol. En general los polímeros biodegradables requieren componentes que aporten características de humectación, plasticidad, lubricación, extensión, conductividad y resistencia, entre otros. Por ello, fue necesario hacer la síntesis de las películas de almidón a través de la adición de algunos agentes (glutaraldehído, glicerol y polietilenglicol). De otra parte, se sintetizó las películas de polipirrol (PPy) con los contra aniones (pTS) e (IC), y se realizó la caracterización electroquímica de cada uno de estos materiales empleando las técnicas electroquímicas de voltametría cíclica (VC) y espectroscopía de impedancia electroquímica (EIE), para evaluar su desempeño como materiales con potencial para ser utilizados en un acumulador electroquímico de carga. 


\section{PARTE EXPERIMENTAL}

El almidón de yuca fue extraído mediante el método tradicional, que comprende las operaciones básicas de lavado, pelado, rallado, decantación, secado y molienda. La calidad del producto fue evaluada mediante la norma técnica ISO 6647. El glicerol (GLY), glutaraldehído (GLU), polietilenglicol (PEG) y perclorato de litio (LP) fueron adquiridos de Sigma-Aldrich. Para la preparación de las biopelículas fue necesario adecuar el agua aumentándole su pH hasta 9. La adición de GLY, GLU, PEG y LP, se realizó a temperatura ambiente y agitación constante. Las disoluciones fueron calentadas a una temperatura controlada de $75 \pm 1,0{ }^{\circ} \mathrm{C}$ durante 15 min y luego fueron vertidas en bandejas de teflón, para calentarlas en una estufa a $70^{\circ} \mathrm{C}$ durante 48 h. En esta investigación se empleó tres mezclas diferentes cambiando la concentración de LP ( 0 gr, 1,5 gr, 3,0 gr), debido principalmente a que estas proporcionan excelentes propiedades eléctricas descritas en investigaciones anteriores ${ }^{4}$.

Para la síntesis electroquímica del polipirrol (electrodos para el acumulador), se empleó la cronoamperometría sobre una placa de acero inoxidable pulido de dimensión $2 \mathrm{~cm} \mathrm{x} 2 \mathrm{~cm}$. Las películas de polímero se electrodepositaron sobre estas placas. Las películas de PPy/pTS fueron sintetizadas a partir de una disolución de $0,1 \mathrm{~mol} \mathrm{l}^{-1}$ de pirrol y $0,1 \mathrm{~mol} \mathrm{l}^{-1}$ de pTS. El proceso de polimerización se llevó a cabo a temperatura ambiente a un potencial de 0,8 $\mathrm{V}$ durante $5000 \mathrm{~s}$, empleando un electrodo de referencia de calomel $\mathrm{KCl}$ saturado, y como contraelectrodo y sustrato se utilizó placas de acero inoxidable de $2 \mathrm{~cm}$ x $2 \mathrm{~cm}$.

Una vez sintetizadas las películas delgadas de PPy, éstas fueron lavadas con agua ultrapura (Milli-Q) y luego introducidas en una solución $0,1 \mathrm{~mol} \mathrm{l}^{-1} \mathrm{LiClO}_{4}$ para la polarización de los electrodos. Este proceso fue llevado a cabo a un potencial de $0,5 \mathrm{~V}$ durante un tiempo de 600 s, convirtiendo la película de PPy/pTS en una película totalmente oxidada (cátodo). Por otro lado, las películas de PPy con índigo carmín (PPy/IC) fueron sintetizadas mediante un procedimiento similar al descrito para las películas de PPy/pTS, con la única diferencia de que para estas películas la polarización fue llevada a cabo a -1,3 V durante un tiempo de 600 $\mathrm{s}$, lo que nos permitió tener una película totalmente reducida (ánodo).

Las celdas utilizada para la caracterización de las películas, tanto para la voltametría cíclica como para la espectroscopía de impedancia, consistió en una celda para muestras sólidas compuestas por dos láminas de acero inoxidable de $2 \mathrm{~cm}$ x $2 \mathrm{~cm}$, fijadas en placas de acrílico que se cierran por un sistema de tuercas. Para realizar las medidas, las películas fueron cortadas en muestras de $1 \mathrm{~cm} \times 1 \mathrm{~cm}\left(1 \mathrm{~cm}^{2}\right)$ y colocadas entre las láminas de acero del porta muestras. Para la técnica de VC se escogió una ventana de potencial de $-2 \mathrm{~V}$ a $2 \mathrm{~V}$, y una velocidad de barrido de $100 \mathrm{mVs}^{-1}$. Para las medidas de EIE se utilizó un rango de frecuencia de $100 \mathrm{kHz}$ a $100 \mathrm{mHzy}$ una amplitud de $10 \mathrm{mVrsm}$. Todos los potenciales fueron medidos en relación con el potencial de circuito abierto (OCP) de $-0,022 \mathrm{~V}$. Con el fin de mejorar la resolución de los procesos rédox en los voltamogramas; éstos fueron analizados mediante un tratamiento de señales conocido como "Nonlinear curve Fitting", lo cual permitió ajustar la posición y la anchura de los picos. 


\section{RESULTADOS Y DISCUSIÓN}

\section{Preparación de las películas de almidón}

Durante el proceso de obtención de las películas delgadas de almidón, fue observado que las condiciones ambientales de humedad afectan significativamente a las películas de almidón, haciéndolas más flexibles a mayor humedad en los primeros 3 días. En el momento en que las películas de almidón son preparadas tienen un comportamiento flexible, que se le atribuye a la adición de plastificante (glicerol), debido a la capacidad que tiene éste de reducir los enlaces de hidrógeno entre las cadenas de glucosa del almidón y de aumentar el espacio entre las moléculas ${ }^{9,10}$.

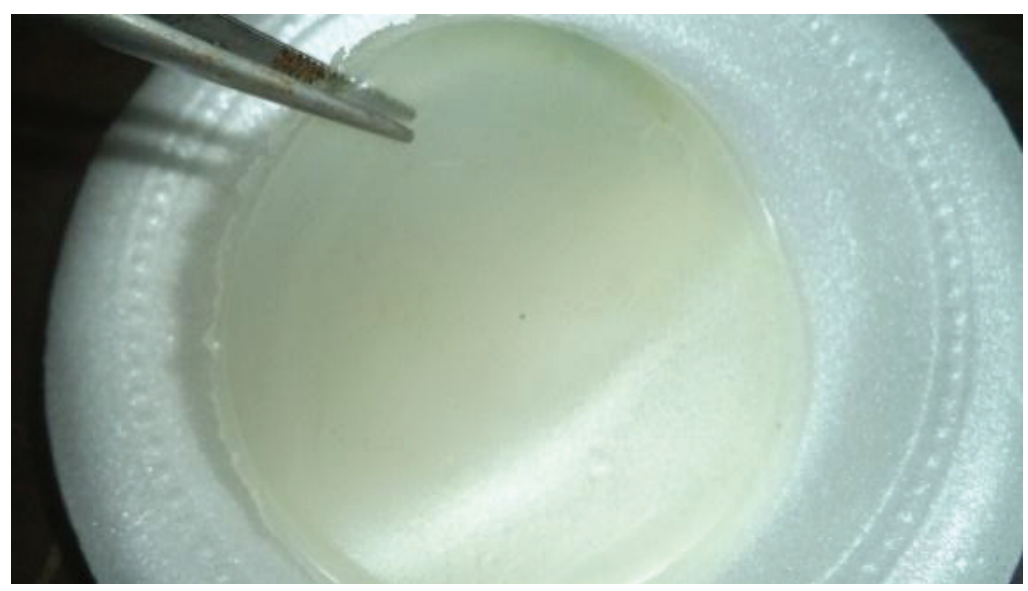

Figura 1. Fotografía de una película de almidón de yuca.

Una vez preparadas las biopelículas presentan una apariencia transparente como se puede apreciar en la figura 1, pero a partir del tercer día éstas toman un color amarillento y opaco, además de que exudan un líquido sobre su superficie; esto es, quizás, debido al exceso en la concentración de plastificante, lo cual provoca la separación de las fases y la exclusión física de dicha sustancia ${ }^{11}$.

\section{Síntesis electroquímica de las películas de polipirrol}

Durante el proceso de electropolimerización de las películas delgada de PPy, se generó curvas cronoamperométricas, las cuales son típicas en este tipo de procesos. En la figura 2, se presenta la curva de electropolimerizacióncronoamperométrica de una película de PPy/ pTS; en esta se observan tres zonas; una primera zona (zona I) que corresponde al aumento abrupto de la corriente atribuido a procesos como la oxidación de los electrodos metálicos, debido al medio acuoso y a la oxidación de los monómeros. Un segundo proceso (zona II) donde tiene lugar el fenómeno de nucleación del polímero (adherencia del polipirrol sobre el electrodo de acero) y finalmente un tercer proceso debido al crecimiento gradual de la película de polipirrol (zona III). 


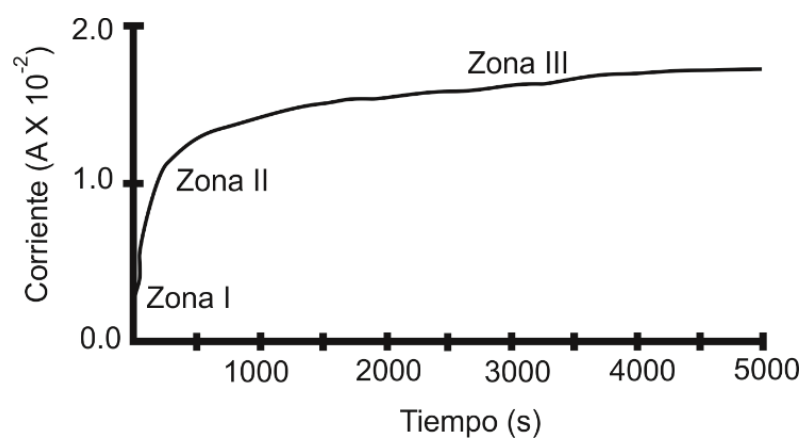

Figura 2. Cronoamperograma de la electropolimerización de polipirrol (PPy) dopada con pTS (PPy/pTS) a un potencial de $0,8 \mathrm{~V}$ durante $5000 \mathrm{~s}$.

Los espesores para las películas de pirrol dopadas con pTS fueron aproximadamente de $48.5 \mu \mathrm{m}$, mientras que para las películas de pirrol dopadas con IC los espesores fueron aproximadamente a 47,68 $\mu \mathrm{m}$. Los espesores se determinaron a partir de la siguiente ecuación. (1), correspondiente a la derivada de la primera ley de Faraday:

$$
d=\frac{Q M}{2 F \rho M}
$$

Donde Q es la carga especifica por unidad de área polimerizada, $\mathrm{M}$ es la masa molar del pirrol $\left(67 \times 10^{-3} \mathrm{~kg} \mathrm{~mol}^{-1}\right)$, F la constante de Faraday y $\rho \mathrm{M}$ la densidad másica del pirrol (1500 $\mathrm{kgm}^{-3}$ ). Esto implica una proporcionalidad directa entre la carga consumida y la cantidad de polímero depositado, asumiendo que en el proceso está implicado un mecanismo de 2 electrones de la molécula de monómero ${ }^{12}$ y que la eficiencia de la corriente es $100 \%$.

Al momento de la electropolimerización, el polipirrol depositado se va oxidando a medida que crece más polímero sobre él, es decir, después de la electropolimerización el polipirrol obtenido queda en un estado levemente oxidado. El polipirrol como material electroactivo puede ser oxidado o reducido alcanzando diferentes estados rédox. Esta propiedad es importante en la aplicación de este material como electrodo, debido a que permite oxidarlo o reducirlo pudiendo ser utilizado como ánodo o cátodo. Al mantener la película de polipirrol en un potencial negativo de voltaje, ésta es reducida; en el caso de aplicar un potencial positivo de voltaje, es oxidada. Por ello, al aplicar potenciales negativos a las películas de $\mathrm{PPy} / \mathrm{IC}$, los cronoamperogramas comienzan con corrientes negativas y van evolucionando hacia corrientes cero, momento en el cual indica que la película se ha reducido totalmente. Caso contrario para los potenciales positivos aplicados a las películas de PPy/pTS, en este caso los cronoamperogramas comienzan con corrientes positivas y van aumentando hasta llegar a una corriente cero, lo que indica el estado de oxidación completo de la película. La utilización de esta propiedad ha sido reportada en la literatura por otros autores para generar baterías a partir de polipirrol y los dopante IC y $\mathrm{pTS}^{13,14}$. 


\section{Análisis de VC y EIE de las películas de almidón de yuca}

La caracterización voltamétrica se empleó para estudiar el comportamiento electroquímico de las películas conductoras de almidón; es decir, para observar los ciclos de oxidación y reducción que se presentan. El análisis electroquímico de las películas delgadas de almidón fue realizado para cada una de las mezclas utilizadas, para las películas de almidón preparadas como blanco (con solo un componente) y las sustancias puras. Como resultado de este estudio, se observó una ausencia de electroactividad con movimiento de cargas (no farádico) en las películas preparadas solo con almidón (figura 3a). Cuando se prepararon películas de almidón con alguno de los componentes (glicerol, polietilenglicol o perclorato de litio) las voltametrías registradas mostraron un proceso rédox en las películas. En la figura $3 \mathrm{~b}$ se presenta como ejemplo la repuesta de una película de almidón con glicerol. Sin embargo, cuando se hizo este mismo análisis sobre cada uno de los componentes de manera independiente, no se observó ningún tipo de actividad rédox.

Este comportamiento puede ser debido a que la adición de componentes en las películas delgadas de almidón genera una reacomodación de las cadenas poliméricas produciendo posibles cambios en la estructura y por ende en sus propiedades electroquímicas ${ }^{3}$.

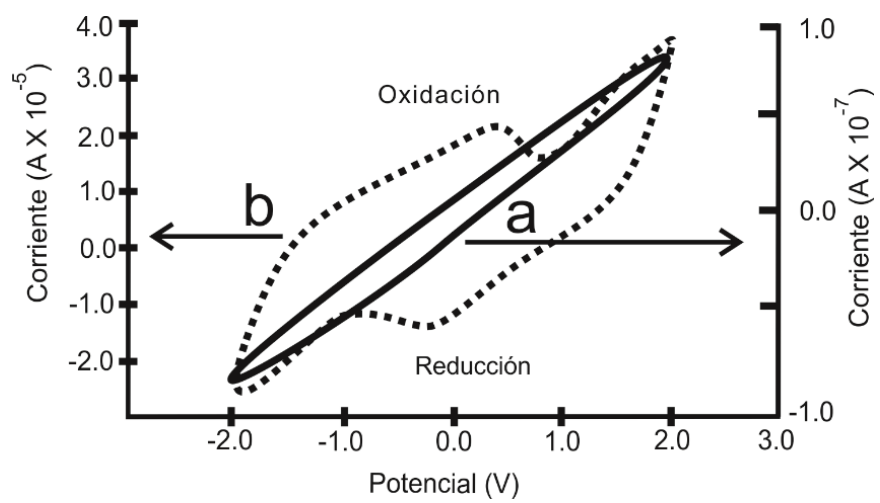

Figura 3. Voltamogramas de películas de almidón: (a) sin aditivos y (b) almidón más glicerol.

En los voltamogramas de las películas delgadas de almidón elaboradas con todos los componentes, se observó que a medida que aumenta la cantidad de éstos, los picos correspondientes a los procesos de oxidación y reducción se registran más agudos, quizás debido a un aumento de las interacciones entre las moléculas de los aditivos y la cadena polimérica de almidón.

Para analizar la estabilidad electroquímica de las películas, se evaluó su comportamiento voltamétrico durante 10 días seguidos y se observó cambios en su comportamiento hasta el sexto días. Los voltamogramas registrados en los primeros días muestran picos anchos y poco definidos, mientras que a partir del sexto día en adelante, las curvas registradas no 
presentaron cambios (medidos hasta 10 días); los picos se presentan mejor definidos y con mayor intensidad. Este fenómeno puede ser debido a que las películas recién sintetizadas pasan por un proceso de reacomodamiento de carga en la matriz polimérica, lo cual genera una mayor eficiencia en el transporte de éstas, lo que explica la mayor intensidad en las señales y el cambio de la electroactividad (figura 4).

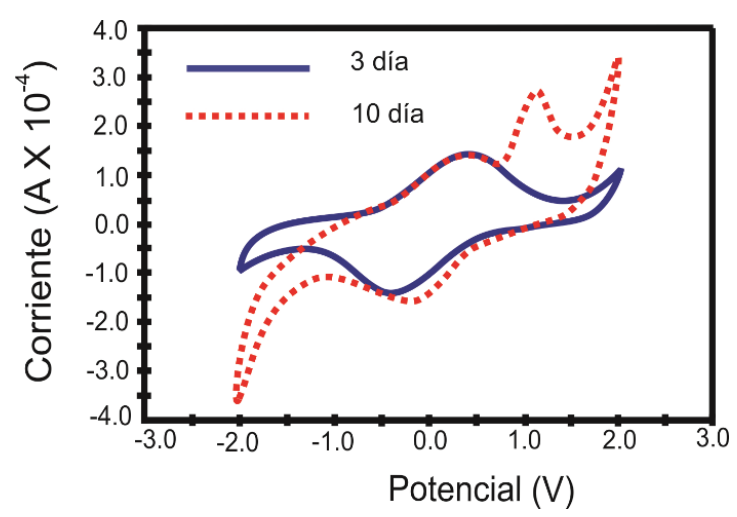

Figura 4. Voltamograma de películas de almidón con plastificante medidos en diferentes días de haber sido sintetizadas.

Por otra parte, al realizar el análisis de EIE a las películas preparadas con almidón y sin aditivos, se observó una alta resistencia eléctrica $(6,50$ x $106 \mathrm{ohm})$. Mientras que las películas preparadas con todos los aditivos (glicerol, glutaraldehído y polietilenglicol), mostraron una disminución de la resistencia eléctrica en dos órdenes de magnitud $\left(8,69 \times 10^{4} \mathrm{ohm}\right)$ con respecto a la preparada sin ellos. A partir de los espectros de impedancia se determinó los datos de resistencia utilizando para ello el intercepto de las curvas en el eje Zre, debido a que la parte real de $\mathrm{Z}$ es simplemente $\mathrm{R}$. En las películas preparadas con almidón, aditivos y perclorato de litio la resistencia eléctrica bajó cuatro órdenes de magnitud respecto a la que contiene sólo almidón $\left(1,59 \times 10^{2} \mathrm{ohm}\right)$; las conductividades de estas películas varió en un rango de $1,28 \times 10^{-3} \mathrm{Scm}^{-1}$ a $8,61 \times 10^{-4} \mathrm{~S} \mathrm{~cm}^{-1}$. Este cambio significativo de la conductividad eléctrica es debido a que los aditivos mejoran las propiedades de transporte de carga en la matriz polimérica y en el caso del perclorato de litio, la naturaleza iónica de esta sal hace que la conductividad se incremente drásticamente?

Adicionalmente, el análisis de EIE mostró algunas diferencias en los mecanismos de transporte de carga de las distintas películas estudiadas. Las películas preparadas con aditivos, mostraron un comportamiento semejante a las sintetizadas sólo con almidón. En la figura 5 se presenta el comportamiento de la película de almidón con los aditivos (figura 5a) y el de la película de almidón con aditivos y perclorato de litio (figura 5b). Las películas preparadas con almidón y aditivos (figura 5a), mostraron un mecanismo de tipo resistivo, compuesto por una resistencia debida al paso de electrones del sustrato a la película y un elemento de fase constante producto, posiblemente, de la formación de capacitancia generadas por 
rugosidades o superficies heterogéneas en la película $\mathrm{R}(\mathrm{Q})$. Este cambio en el mecanismo de transporte es evidencia de que la adición de sal de litio aumenta la conductividad y la cinética de movimiento de cargas en la matriz polimérica debido a la presencia de iones. Sin embargo, las películas preparadas con aditivos y iones de litio presentaron un comportamiento drásticamente diferente, mostrando un mecanismo formado por una resistencia; debida al paso de electrones del sustrato a la película, conectada en seria a una sistema capacitivo en paralelo con una resistencia y una resistencia de Warburg; $\mathrm{R}(\mathrm{C}[\mathrm{RW}])$, producidas por las resistencias de paso de las cargas a través de la matriz polimérica (figura 5b) y la acumulación de carga en las cadenas poliméricas.

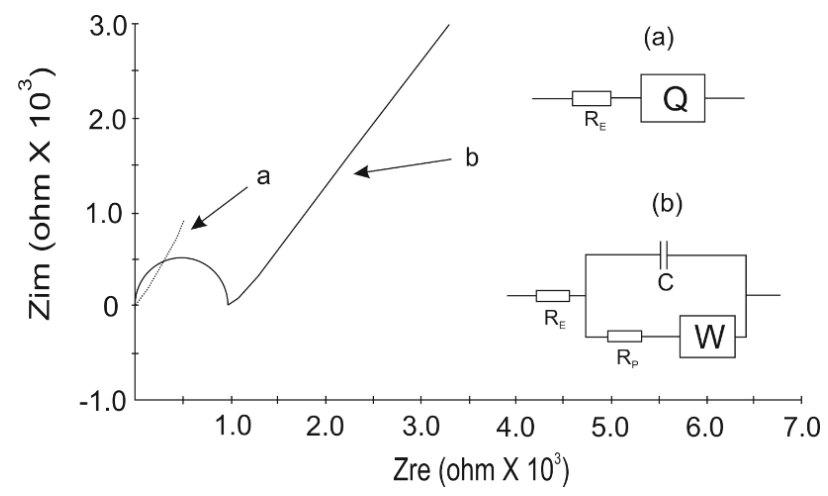

Figura 5. Diagramas de Nyquist y Circuito Equivalente: (a) película de almidón con aditivos y (b) película de almidón con aditivos y litio.

\section{Análisis de VC y EIE de las películas de polipirrol}

En cuanto al análisis de VC de los electrodos de polipirrol realizados en una solución 0,1 $\mathrm{M}$ de $\mathrm{LiClO}$, se pudo observar claramente dos procesos rédox, tanto para las películas de PPy/pTS (figura 6 a) y las de PPy/IC (figura 6 b). En ambos casos se observa un proceso de oxidación en la onda anódica.
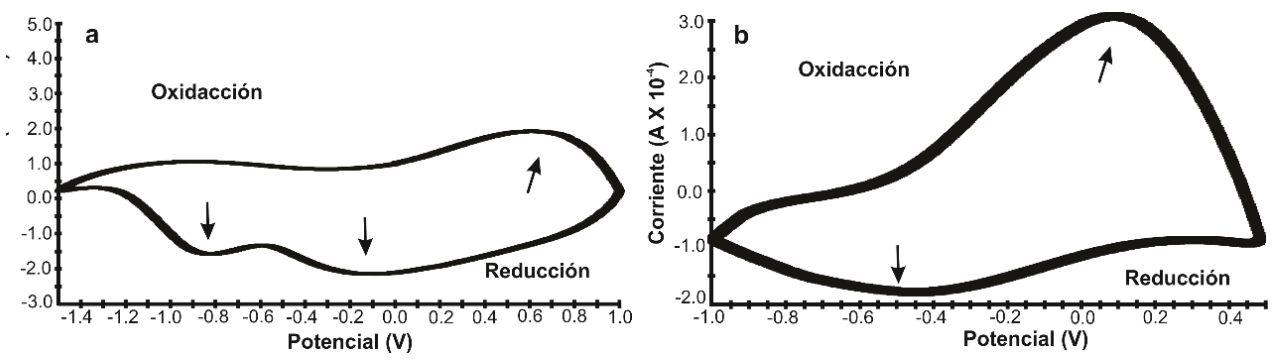

Figura 6. Voltamograma película de a)PPy/pTSy b) PPy/IC. 
En la onda catódica de la película delgada de PPy/pTS, se puede observar los dos procesos bien definidos y que corresponden a dos procesos de reducción de PPy reportados en la literatura ${ }^{9}$. De la misma forma, en los voltamogramas de las películas de PPy/IC la onda anódica muestra un proceso de reducción de las cadenas de polipirrol. En la zona catódica, en ambos casos se observó picos anchos, debidos a la oxidación de las películas. La naturaleza de los procesos electroquímicos que tienen lugar durante la oxidación/reducción de las películas de pirrol no ha sido aún determinado, aunque se puede interpretar con el modelo del polarón/bipolarón, según el cual la oxidación parcial de la cadena polimérica conduce a la formación de un polarón, es decir, una asociación de un electrón no localizado y una carga positiva. Al eliminar un segundo electrón (segunda oxidación) de la misma sección de cadena polimérica, puede formarse un segundo polarón independiente del ya existente, o puede ocurrir que se elimine el electrón deslocalizado para formar un dicatión o bipolarón. Durante el primer proceso de oxidación/reducción, que tiene lugar a más bajo potencial, el anión dopante se encuentra atrapado en la matriz polimérica, de modo que para mantener la electroneutralidad, el polímero intercambia cationes con el medio. En el segundo proceso de oxidación/reducción de la película, la matriz polimérica intercambia aniones con el medio y así mantiene su electroneutralidad9. El mecanismo propuesto se puede representar de la siguiente manera.

$$
\begin{aligned}
& {\left[\mathrm{PPy}\left(X^{-} A^{+}\right)\right] \leftrightarrow\left[\mathrm{PPy}^{+} X^{-}\right]+A+e^{-}} \\
& {\left[\mathrm{PPy}^{+} X^{-}\right]+\mathrm{Y}^{-} A^{+} \leftrightarrow\left[\mathrm{PPy}^{2} \mathrm{X}^{-} \mathrm{Y}^{-}\right]+A^{+}+e^{-}}
\end{aligned}
$$

Los resultados de EIE realizado a las películas delgadas de pirrol y que se muestran en la figura 7, describe un mecanismo de transferencia de iones equivalente a un circuito resistivo capacitivo para el cátodo y con tendencia más capacitiva para el ánodo. Lo que comprueba que ambos materiales son apropiados para ser empleado como electrodos del acumulador.

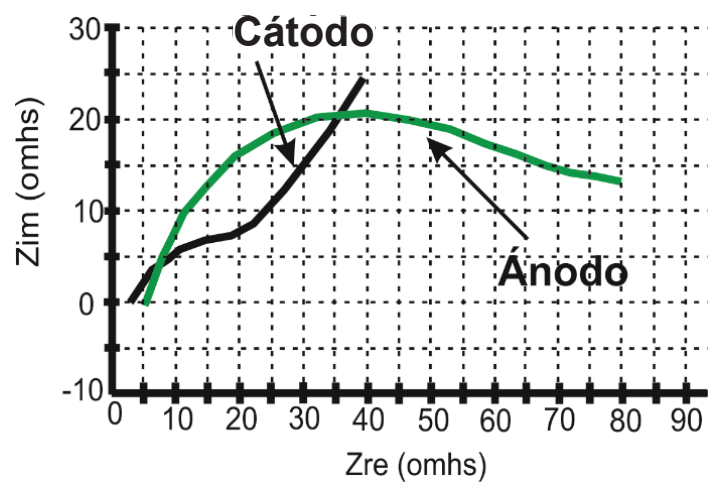

Figura 7. Espectros de EIE de las películas de PPy/pTS (cátodo) y PPy/IC (ánodo). 


\section{CONCLUSIONES}

Mediante el desarrollo de este trabajo, se obtuvo películas poliméricas delgadas con propiedades conductoras que variaron en un rango de $1,28 \times 10^{-3} \mathrm{Scm}^{-1}$ a $8,61 \times 10^{-4} \mathrm{Scm}^{-1}$ clasificándolo de acuerdo a la escala de conductividad como materiales semiconductores. Por otro lado, presentaron comportamiento estable y homogéneo.

Para los ensayos de VC realizados a las películas delgadas de almidón se detalla la presencia de procesos rédox (oxidación y reducción) que permite la acumulación de carga en el dispositivo armado; estos picos de potenciales se ven más definidos y estables a los 10 días de su elaboración. Con esto se garantiza la buena funcionalidad de estas películas como electrolito sólido polimérico del acumulador. De la misma manera, los mecanismos de trasferencia de carga cambian de resistivo/capacitivo a netamente resistivo según la composición del biopolímero, y se debe a la movilidad iónica del litio en la matriz del polímero. Los electrodos de pirrol dopado con pTS e IC al igual que las películas delgadas de almidón, presentan propiedades rédox que permite su oxidación y reducción reversible lo que muestra su excelente comportamiento como electrodos.

\section{AGRADECIMIENTO}

Los autores agradecen el soporte financiero brindado por la Universidad Pontificia Bolivariana - Montería y la Gobernación de Córdoba bajo el convenio No. 753 -2013 "Desarrollo de Integración Tecnológica de Recursos Energéticos Renovables en Sistemas Productivos Agrícolas y Agroindustriales Montería, Córdoba, Caribe” BPIN No. 2012000100026. Y a CONICYT-PCHA/Doctorado Nacional/2014-63140015

\section{BIBLIOGRAFÍA}

1. J. Padilla, R. Garcia, A.J. Fernandez, A. Urbina, "Polímeros conductores Su papel en un desarrollo energético sostenible", Primera edición, Editorial Reverté, Barcelona, España, 2012, 51.

2. L. Sultana, M.M. Rahman, J. Wang, C. Wang, G.G. Wallace, H.K. Liu, Electrochim. Acta, 2012; 83: 209.

3. A. Arrieta, M. Palencia, Rev. LatinAm. Metal. Mat., 2016; 36: 1.

4. A.A. Arrieta, P. Gañan, S. Márquez, R. Zuluaga, J. Braz. Chem. Soc, 2011; 22:1170.

5. Y.V. Garcia, P.B. Zamudio, L.A. Bello, C.A. Romero, J. Solorza, Revista Iberoamericana de Polímeros, 2011; 3: 125.

6. F. Gray, "Solid Polymer Electrolytes, Fundamental and Technological Applications", University of St. Andrews, VCH Publishers, 1991, 92.

7. J.M. Prabaharan, S. Radhakrishna, Solid State Ionics, 1997; 104: 267.

8. F.D. Eramo, T.F. Otero, Cerámica y Vidrio, 2000; 39: 377.

9. P.M. Forssell, P. Myllarinen, G.K. Moates, R. Parker, Carbohydr. Polym., 1999; 39: 43.

10. P. Myllarinen, R. Seppala, P. Forssell, Carbohydr. Polym., 2002; 50: 355.

11. R. Velasco, M. Enriquez, A. Torres, L. Palacios, J. Ruales, Biotecnología en el sector Agropecuario y Agroindustrial, 2012; 10: 152. 
12. A.A. Arrieta, R.L. Vieira, J. Chil. Chem. Soc., 2006; 51: 971.

13. I. Sultana, M.M. Rahman, J. Wang, G. Wang, G.G. Wallace, H.L. Kun, Solid State Ionics, 2012; 215: 29.

14. I. Sultana, M.M. Rahman, S.Li, J. Wang, C. Wang, G.G. Wallace, H.L. Liu, Electrochim. Acta, 2012; 60: 201. 